\title{
LA-UR-21-27851
}

Approved for public release; distribution is unlimited.

Title: $\quad$ Neutron Detection Efficiency Measurements of the Domino Detector for the NEMO Experiment

Author(s): $\quad$ Young, Christian Xavier

Bartlett, Kurtis David

Smith, Karl

Barney, Jonathan Elijah

Intended for: Keepin Program end-of-summer presentations

Issued: 2021-08-05 
Disclaimer:

Los Alamos National Laboratory, an affirmative action/equal opportunity employer, is operated by Triad National Security, LLC for the National Nuclear Security Administration of U.S. Department of Energy under contract 89233218CNA000001. By approving this article, the publisher recognizes that the U.S. Government retains nonexclusive, royalty-free license to publish or reproduce the published form of this contribution, or to allow others to do so, for U.S. Government purposes. Los Alamos National Laboratory requests that the publisher identify this article as work performed under the auspices of the U.S. Department of Energy. Los Alamos National Laboratory strongly supports academic freedom and a researcher's right to publish; as an institution, however, the Laboratory does not endorse the viewpoint of a publication or guarantee its technical correctness. 


\section{Neutron Detection Efficiency Measurements of the Domino Detector for the NEMO Experiment}

Christian Young $^{1,2}$, Dr. Kurtis Bartlett ${ }^{1}$, Dr. Karl Smith ${ }^{1}$, Dr. Jonathan Barney ${ }^{1}$

${ }^{1}$ Los Alamos National Laboratory Intelligence and Space Research Space Science and Applications

2University of Tennessee Tickle College of Engineering Department of Nuclear Engineering 


\section{Summer Fun}
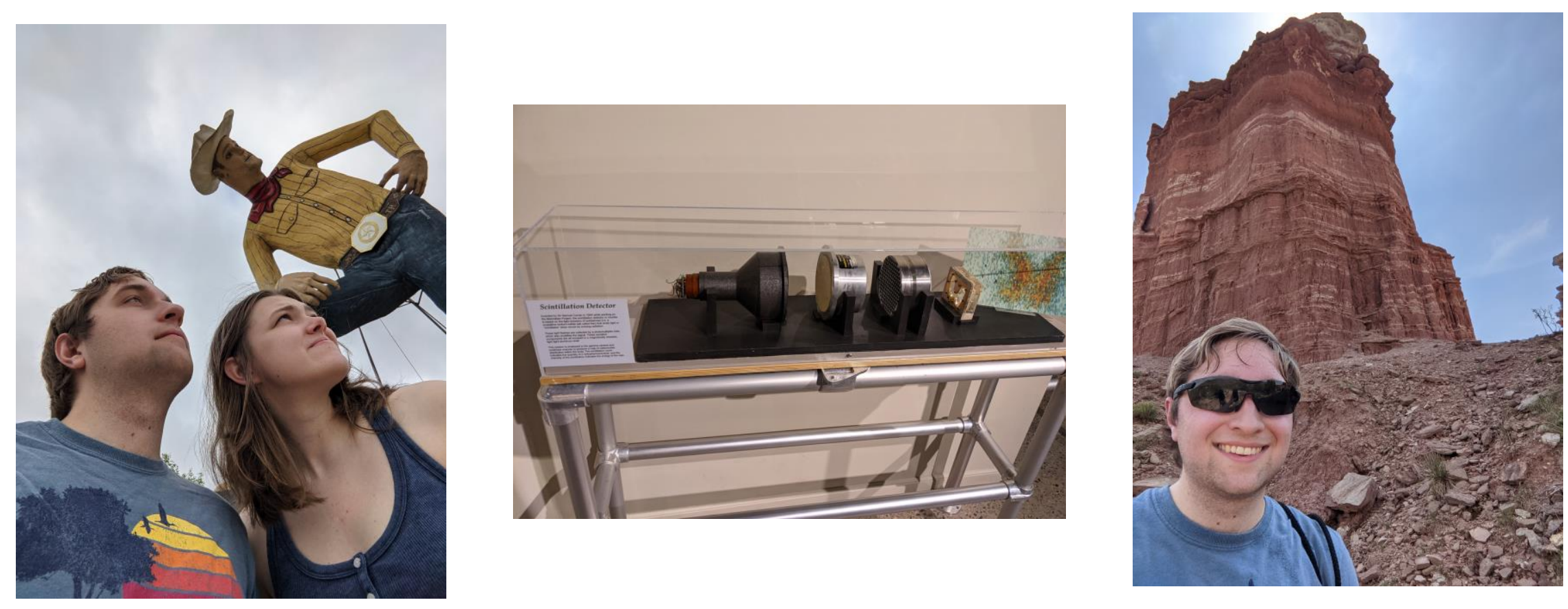


\section{Christian Young (ISR-1)}

- Educational Background

- Purdue University, 2020

- B.S. Nuclear Engineering

○ University of Tennessee, 2025?

- Ph.D. Nuclear Engineering

- Intelligence and Space Research (ISR)

- Space Science \& Applications (ISR-1)

○ Dr. Kurtis Bartlett, Dr. Karl Smith
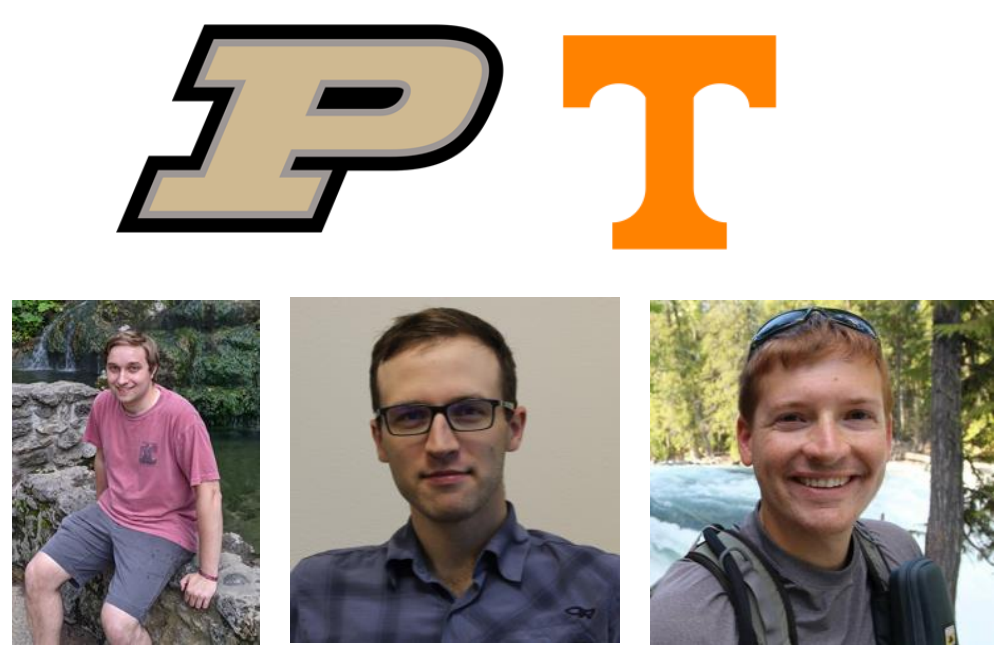

- Research

- Neutron detector efficiencies for moon orbit mission

o Work at UTK: Neutron detection and radiography 


\section{Research Overview and Motivation}

- Neutron lifetime measurements disagree - Plot from the Particle Data Group

- Differences stem from "beam" vs "bottle"

- New idea: space as a form of containment

o Thermal neutrons from moon's surface

- Neutron-lifetime Experiment in the Moon's Orbit

- CubeSat ride-along

- Domino neutron detector on board

- Need to validate the detector's efficiency

o RDT data only based on MCNP simulations
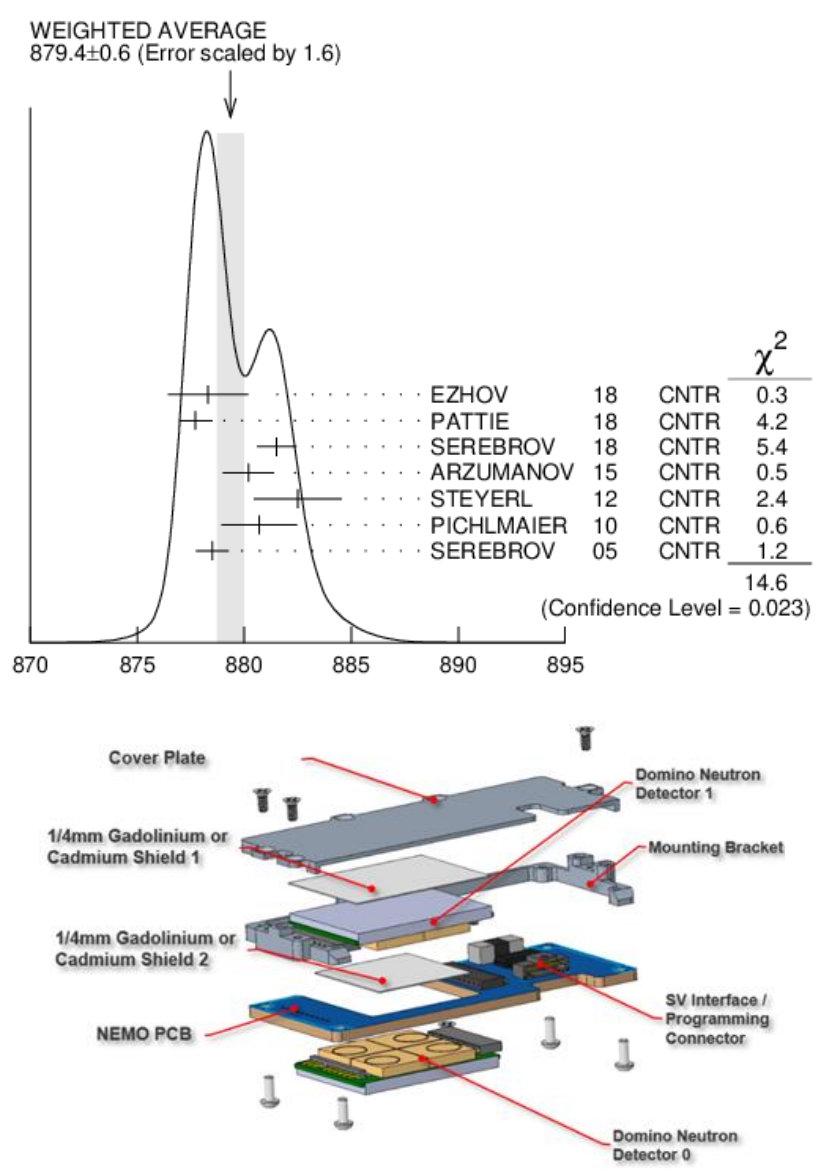


\section{Research Approach}

- Combined simulation and physical approach

o MCNP: Energy-dependent flux

o Physical: True count rates

- Take several measurements

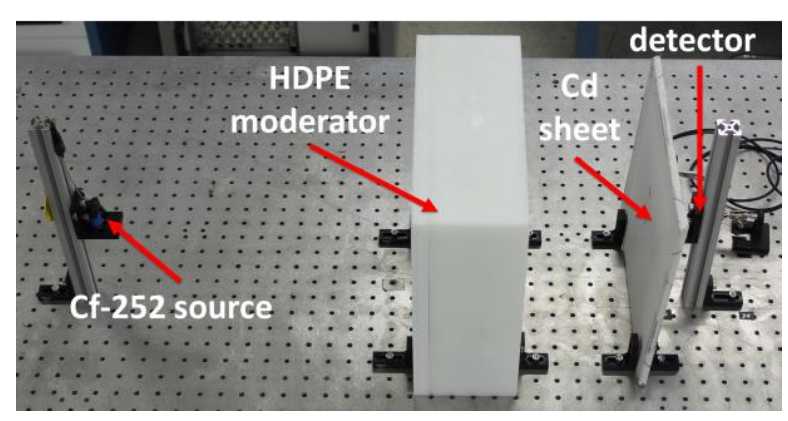

- Variable moderator thicknesses

- Cf-252 source

- Iterative unfolding process to extract efficiencies

- Maximum-likelihood expectation-maximization

o Monte Carlo methods for uncertainty

$$
\left[\begin{array}{cccc}
x_{1,1} & x_{1,2} & \cdots & x_{1, m} \\
x_{2,1} & \ddots & & \vdots \\
\vdots & & \ddots & \vdots \\
x_{n, 1} & \cdots & \cdots & x_{n, m}
\end{array}\right]\left[\begin{array}{c}
\varepsilon_{1} \\
\varepsilon_{2} \\
\vdots \\
\varepsilon_{m}
\end{array}\right]=\left[\begin{array}{c}
T_{1} \\
T_{2} \\
\vdots \\
T_{n}
\end{array}\right]
$$




\section{Summary of Results}

- Figure shows calculated neutron detection efficiencies up to $1 \mathrm{MeV}$

- Agreement with RDT efficiencies

- Improvements can be made

- Improved physical measurements

- Reduced environmental impact

- Longer count times

- LANSCE

- Time-of-flight measurements

o Finer binning
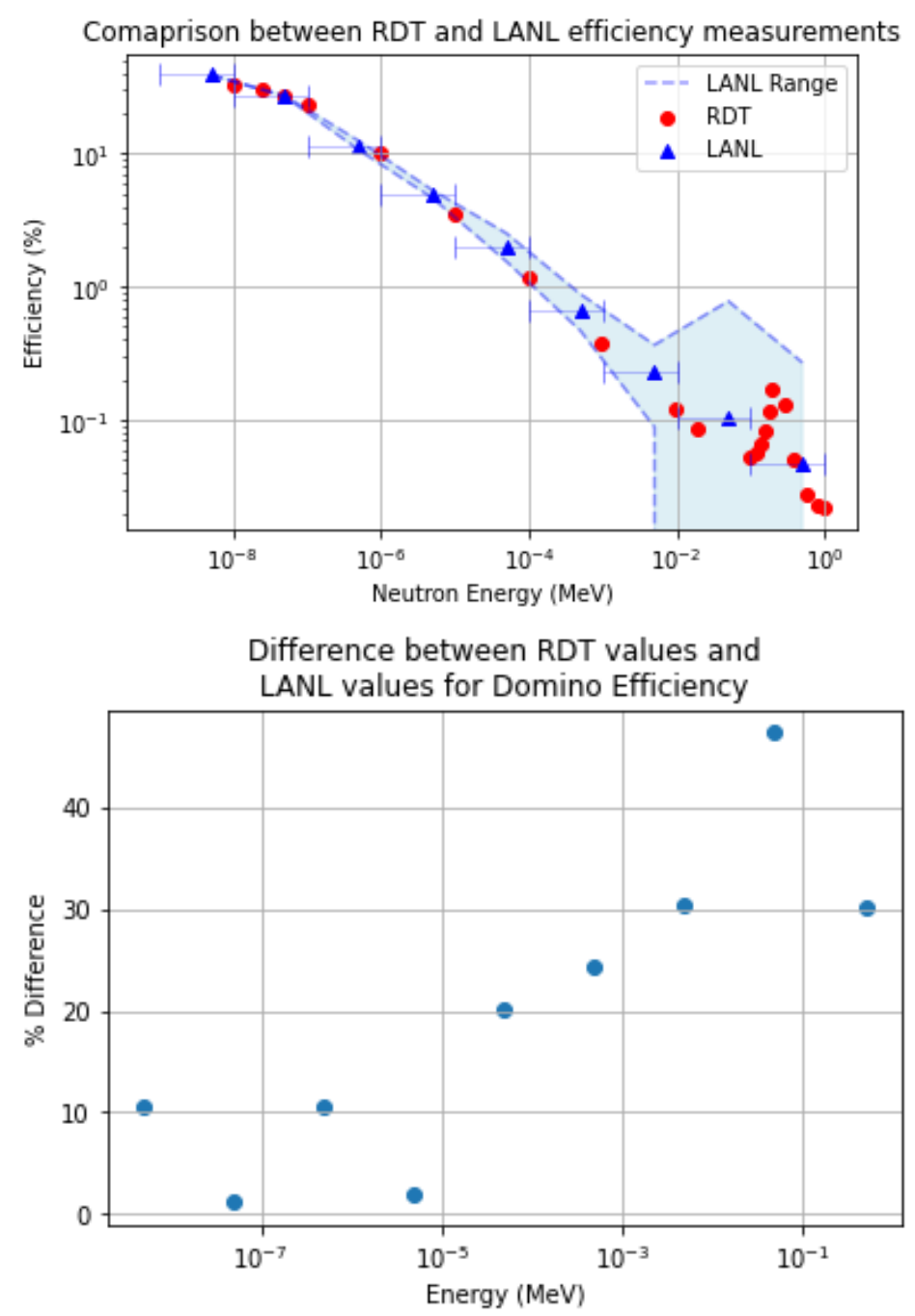